\title{
Vanadium supersaturated silicon system: a theoretical and experimental approach
}

\author{
Eric Garcia-Hemme ${ }^{1} \odot$, Gregorio García ${ }^{2,3} \odot$, Pablo Palacios ${ }^{2,4}$, \\ Daniel Montero ${ }^{1}$, Rodrigo García-Hernansanz ${ }^{1}$, Germán Gonzalez-Diaz ${ }^{1}$ \\ and Perla Wahnon ${ }^{2,3}$ \\ 1 Departamento de Física Aplicada III, Univ. Complutense de Madrid, Ciudad Universitaria, 28040 \\ Madrid, Spain \\ ${ }^{2}$ Instituto de Energía Solar, ETSI Telecomunicación, Universidad Politécnica de Madrid, 28040 Madrid, \\ Spain \\ ${ }^{3}$ Departamento de Tecnología Fotónica y Bioingeniería, ETSI Telecomunicación, Universidad \\ Politécnica de Madrid, Ciudad Universitaria, s/n, 28040 Madrid, Spain \\ ${ }^{4}$ Departamento de Física aplicada a las Ingenierías Aeronáutica y Naval, ETSI Aeronáutica y del Espacio, \\ Universidad Politécnica de Madrid, Pz. Cardenal Cisneros, 3, 28040 Madrid, Spain \\ E-mail: ggmoreno@etsit.upm.es (G García) and eric.garcia@ucm.es (E García-Hemme)
}

Received 11 July 2017, revised 18 September 2017

Accepted for publication 13 October 2017

Published 13 November 2017

\begin{abstract}
The effect of high dose vanadium ion implantation and pulsed laser annealing on the crystal structure and sub-bandgap optical absorption features of V-supersaturated silicon samples has been studied through the combination of experimental and theoretical approaches. Interest in V-supersaturated Si focusses on its potential as a material having a new band within the Si bandgap. Rutherford backscattering spectrometry measurements and formation energies computed through quantum calculations provide evidence that $\mathrm{V}$ atoms are mainly located at interstitial positions. The response of sub-bandgap spectral photoconductance is extended far into the infrared region of the spectrum. Theoretical simulations (based on density functional theory and many-body perturbation in $G W$ approximation) bring to light that, in addition to $\mathrm{V}$ atoms at interstitial positions, Si defects should also be taken into account in explaining the experimental profile of the spectral photoconductance. The combination of experimental and theoretical methods provides evidence that the improved spectral photoconductance up to $6.2 \mu \mathrm{m}(0.2 \mathrm{eV})$ is due to new sub-bandgap transitions, for which the new band due to $\mathrm{V}$ atoms within the Si bandgap plays an essential role. This enables the use of V-supersaturated silicon in the third generation of photovoltaic devices.
\end{abstract}

Keywords: supersaturated silicon, photovoltaic, DFT, GW, RBS, spectral photoconductance

(Some figures may appear in colour only in the online journal)

\section{Introduction}

Nowadays, the global scientific community is making a joint effort to increase the efficiency of renewable energy systems. Particularly in the field of photovoltaics (PV), a third generation of solar cells, based on new concepts that increase device efficiency, is emerging. Solar cells are commonly divided into three main categories (generations). The first generation of solar cells (also called wafer solar cells) is based on crystalline silicon. They are the most commercially predominant due to high efficiencies. Nonetheless, they are relatively expensive to produce. Second generation solar cells, commonly known as thin-film solar cells, are characterized by their potential to reduce material and production cost. Third generation of solar cells aims to considerably improve device efficiencies, with only a small increase in areal costs, hence reducing the cost per peak Watt. Further, third generation solar cells keep the economic and environmental cost advantages of thin-film deposition techniques (i.e. by using thin-film deposition methods and abundant, non-toxic materials) [1, 2]. 


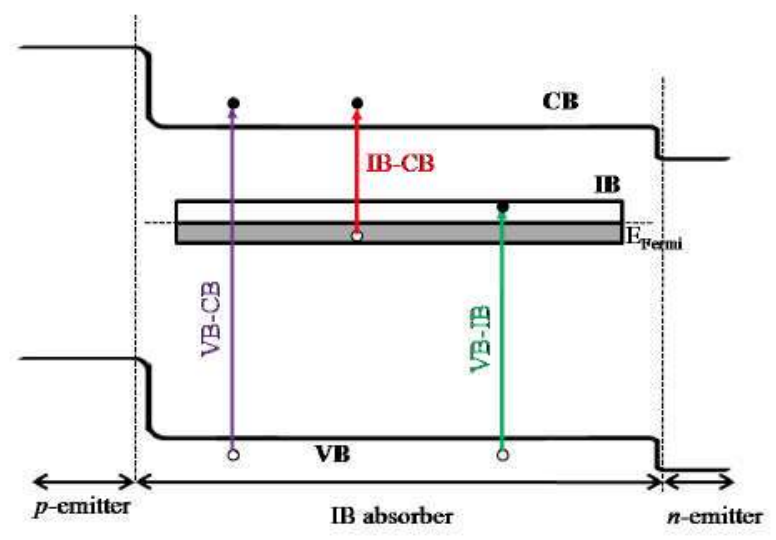

Figure 1. Scheme of the electronic band structure for an IB material located between p- and n-type semiconductors. The three possible electronic transitions for an intermediate band material are also shown.

To achieve improved efficiency, the two main power-loss mechanisms must be bypassed: the inability to absorb photons with energy lower than the bandgap, and the thermalisation of photons with energy higher than the bandgap. To this end, various approaches have been proposed: (i) increasing the number of bandgaps; (ii) capturing carriers before thermalisation; and (iii) multiple carrier pair generation per photon with high energy or single carrier pair generation with multiple photons of low energy. Among these, only those based on the implementation of strategy (i), for instance tandem solar cells, have been developed with efficiencies higher than the Shockley-Queisser limit [1, 2]. Even so, other approaches such as quantum well or impurity band materials have also been widely studied.

The intermediate band solar cell (IBSC), based on an intermediate band (IB) material, is one of the most promising candidates [3]. The concept, called impurity PV device [4], was first proposed by Wolf in 1960 to achieve higher PV efficiency, and was taken up again in 1997 by Luque and Martí, who showed that this type of IBSC could enhance solar efficiency [5]. Since then, a great deal of work has been done toward designing novel IB materials [6,7], describing their physical properties [8], and obtaining practical devices that can demonstrate their operational principle [9]. The IB material is a semiconductor with its classical conduction band (CB) and valence band (VB), which also has a third narrow band of partially filled states within the bandgap (see figure 1). As a result, an IB material would be able to absorb sub-bandgap photons to promote charge carriers from the VB to the IB and from the $\mathrm{IB}$ to the $\mathrm{CB}$ at comparable rates using a wider wavelength range of the solar spectrum, mimicking nature in the so-called Z-scheme that follows a similar strategy in the photosynthesis [10]. This mechanism would increase the sub-bandgap absorption and the solar cell efficiency compared with the Shockley-Queisser limit, which determines the highest efficiency of a single junction solar cell at around $40.7 \%$ for a semiconductor with a $1.1 \mathrm{eV}$ bandgap [11].

Different manufacturing approaches are being explored to obtain an IB material: (i) supersaturated silicon with deep levels [12]; (ii) thin film chalcogenide-based devices, firstly proposed based on theoretical calculations, whose photoluminescence and photocatalyst test demonstrated experimentally, for the first time, the existence of an IB material [13]; (iii) highly mismatched alloys, in which a large anion is substituted by a much lighter one [14]; (iv) quantum dot solar cells [15]. In this work, we deal with the deep level approach, which consists of the introduction of a great amount of deep level impurities in a silicon semiconductor via hyper doping $[16,17]$. At low concentration, deep level impurities produce discrete levels within the host semiconductor bandgap, i.e. localized states that are very effective as recombination centers. However, a different phenomenon takes place when the concentration of impurities overcomes the so-called Mott transition limit $[18,19]$. Due to the closer spatial proximity between impurities, and to the Pauli Exclusion Principle, an energy splitting of the discrete deep levels occurs. This phenomenon leads to a localization-delocalization transition similar to the insulator-metal transition, and thus a band of allowed states with delocalized character is formed within the band gap of the host semiconductor. As a consequence of this new band of allowed states, the non-radiative recombination is reduced and the sub-bandgap absorption increases [20,21]. This concentration limit has been theoretically calculated to be approximately $6 \times 10^{19} \mathrm{~cm}^{-3}[22]$, and experimentally confirmed in Ti [23, 24], V [25], and S [26] supersaturated Si systems. Experimental observations of the lifetime recovery were observed in Ti supersaturated Si material, with an effective carrier lifetime of $3.5 \mu \mathrm{s}$ at a carrier injection level of $10^{16} \mathrm{~cm}^{-3}$ [21]. Among the various transition metals, great effort has been put into the understanding of Ti supersaturated Si materials $[16,24,27,28]$, while theoretical and experimental knowledge regarding the V supersaturated Si system is lacking.

This work combines experimental and computational approaches to infer the main effects of introducing high vanadium concentrations into bulk Si, i.e. V-supersaturated Si material. Rutherford back scattering spectroscopy (RBS) measurements were carried out to obtain information about its structure and composition, while spectral photoconductance yielded information about the sub-bandgap photo-generation processes that take place in the V-supersaturated Si material. Theoretical simulations, based on density functional theory (DFT) and many-body perturbation theory, were carried out to deepen the knowledge of $\mathrm{V}$-implantation from an energetic point of view and the optoelectronic structure of V-supersaturated Si. This work gives us a detailed picture of V-implantation in bulk $\mathrm{Si}$ and its relation with the sub-bandgap photoresponse.

\section{Results and discussion}

To obtain V supersaturated Si material, samples $1 \times 1 \mathrm{~cm}$ in size of float zonen-type $\mathrm{Si}(111)$ with a thickness of $300 \mu \mathrm{m}$ $\left(\rho \approx 200 \Omega \mathrm{cm}, \mu \approx 1500 \mathrm{~cm}^{2} \mathrm{~V}^{-1} \mathrm{~s}^{-1}, n \approx 2.2 \cdot 10^{13} \mathrm{~cm}^{-3}\right.$ at room temperature) were implanted at $32 \mathrm{keV}$ with $\mathrm{V}$ at two different doses $\left(10^{15}\right.$ and $\left.10^{16} \mathrm{~cm}^{-2}\right)$ using a $7^{\circ}$ tilt angle. Subsequently, the implanted samples were processed by pulsed laser melting (PLM) at $1 \mathrm{~J} \mathrm{~cm}^{-2}$ with a $\mathrm{KrF}$ excimer 


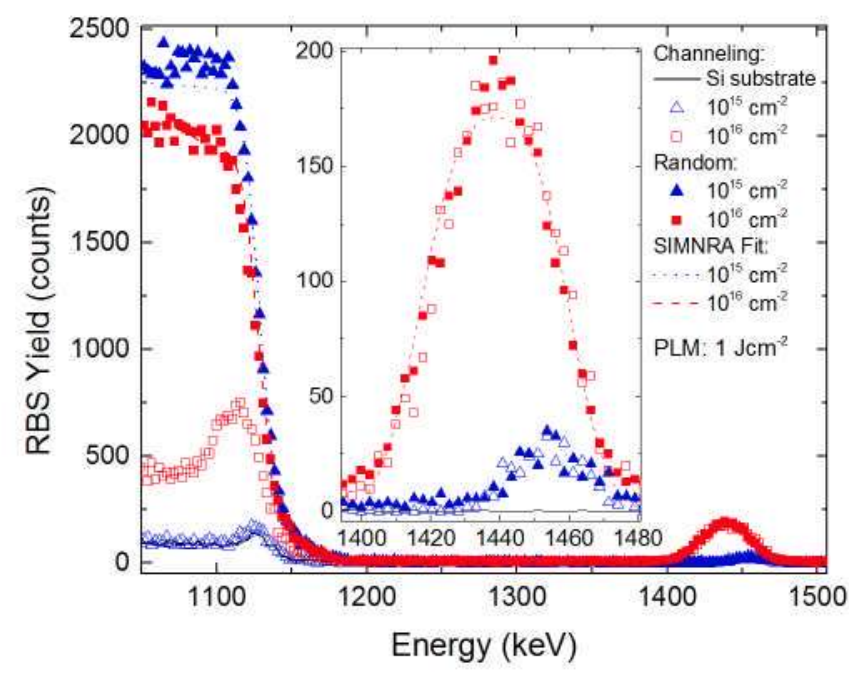

Figure 2. RBS spectra in both channeling and random configurations, for the vanadium supersaturated silicon samples implanted with $10^{15} \mathrm{~cm}^{-2}$ and $10^{16} \mathrm{~cm}^{-2}$ and subsequently pulsed laser melted at $1 \mathrm{~J} \mathrm{~cm}^{-2}$. The channeling spectrum of a Si unimplanted substrate is also presented for comparison. The inset shows details of the spectra centered at the vanadium peak signal (in the energy region from 1390 to $1480 \mathrm{keV}$ ).

laser $(248 \mathrm{~nm})$ at IPG Photonics. An alternative annealing technique to PLM is the flash lamp annealing process with millisecond time duration [29]. This technique allows for upscalable wafer processing. However, it has only proven to be useful for the fabrication of chalcogen-hyperdoped silicon [30]. In the case of transition-metal-hyperdoped silicon, nonhomogeneous hemispherical particles of Si containing $\mathrm{Ti}$ are obtained [31]. Therefore, we decided to use the PLM process for the fabrication of V hyperdoped Si samples. More details on the fabrication process-as well as transmission electron microscopy (TEM) and vanadium concentration depth profiles - of these samples can be obtained from our previous work [25].

Structural information was obtained through RBS. Measurements were carried out in the V supersaturated Si samples ion implanted with doses of $10^{15}$ and $10^{16} \mathrm{~cm}^{-2}$, and subsequently subjected to PLM and processed at $1 \mathrm{~J} \mathrm{~cm}^{-2}$. Figure 2 shows the RBS spectra in both, random and channeling configurations for the $\mathrm{V}$-supersaturated $\mathrm{Si}$ samples at $10^{15} \mathrm{~cm}^{-2}$ and $10^{16} \mathrm{~cm}^{-2}$ doses and subsequently PLM processed at $1 \mathrm{~J} \mathrm{~cm}^{-2}$. Backscattered spectra are analyzed using the SIMNRA simulation program [32]. The low energy side of the spectra, up to $1150 \mathrm{keV}$, shows the contribution of the Si substrate. As observed in the channeling spectra, a peak related to dechanneling effects in the superficial layer emerges around $1120 \mathrm{keV}$ for all the samples. This dechanneling peak is higher for the sample implanted with a higher dose $\left(10^{16} \mathrm{~cm}^{-2}\right)$, whereas it is similar in height for the sample implanted with $10^{15} \mathrm{~cm}^{-2}$ and the untreated $\mathrm{Si}$ substrate. This observation indicates that the sample implanted with the higher dose presents a lower surface Si lattice quality, probably due to a higher number of $\mathrm{Si}$ atoms displaced from their position in the lattice during $\mathrm{V}$ ion implantation. In contrast, the spectrum of the sample implanted with the dose of
$10^{15} \mathrm{~cm}^{-2}$ shows a dechanneling peak (around $1125 \mathrm{keV}$ ) with a very low intensity, clearly similar to that of the silicon substrate, pointing to a very high lattice reconstruction. It has been shown that an average surface roughness of $0.732 \mathrm{~nm}$ appears when silicon samples are annealed at a laser energy density of $1 \mathrm{~J} \mathrm{~cm}^{-2}$ [33]. It is expected that this roughness should not play an important role in the fabrication of the intermediate band solar cell, since the next step is the deposition of an intrinsic a-Si layer of $5 \mathrm{~nm}$ [12], much thicker than the average roughness. Therefore, this deposition process will fully cover and act as a passivation layer of the $\mathrm{V}$-supersaturated $\mathrm{Si}$ layer.

In the high-energy side of the spectra, from 1390 to $1480 \mathrm{keV}$, we can observe the RBS signal of the $\mathrm{V}$ atoms, with different heights and widths depending on the dose implanted. As the implanted dose is increased, the $\mathrm{V}$ signal peak exhibits an increase in the intensity that is related to the higher $\mathrm{V}$ concentration. In addition, a broadening of the peak can be observed as the implantation dose is increased. This effect is related with the $\mathrm{V}$ redistribution produced by the PLM, which is different for the doses of $10^{15}$ and $10^{16} \mathrm{~cm}^{-2}$, being deeper for the higher implanted dose. This profile broadening is likely the result of the increased melt duration during PLM, caused by freezing-point depression due to the large amount of impurity present in the melt [34].

SIMNRA simulations shown in the inset of figure 2 reproduces the RBS spectra and the V peak energy location. From these fittings, an average thickness of V-supersaturated $\mathrm{Si}$ layer can be estimated: $32 \mathrm{~nm}$ for the sample implanted with $10^{15} \mathrm{~cm}^{-2}$ and $92 \mathrm{~nm}$ for the sample implanted with $10^{16} \mathrm{~cm}^{-2}$. Taking into account these thicknesses, we can estimate the $\mathrm{V}$ doses of the samples after PLM process obtained from the SIMNRA fitting: $1.3 \times 10^{15} \mathrm{~cm}^{-2}$ for the sample implanted with $10^{15} \mathrm{~cm}^{-2}$ and $1.5 \times 10^{16} \mathrm{~cm}^{-2}$ for the sample implanted with $10^{16} \mathrm{~cm}^{-2}$.

All these results are in agreement with time of flight secondary ion mass spectrometry (ToF-SIMS) measurements and high-resolution transmission electron microscopy (HRTEM) images of similar samples reported previously [25]. In this sense, ToF-SIMS showed that both samples present a vanadium concentration higher than the theoretical limit to obtain an IB material. Specifically, the sample implanted with the dose of $10^{15} \mathrm{~cm}^{-2}$ exceeds this concentration limit in a layer of $\sim 21 \mathrm{~nm}$, while the sample implanted with $10^{16} \mathrm{~cm}^{-2}$ exceeds it in a layer of $\sim 96 \mathrm{~nm}$. Note that slight differences in the doses are due to the depth resolution of the RBS measurements, which is lower in comparison with the ToF-SIMS measurements $(5-10 \mathrm{~nm})$. HR-TEM images showed a single crystal perfect lattice reconstruction after the PLM process for the sample implanted with $10^{15} \mathrm{~cm}^{-2}$. The sample implanted with the highest dose of $10^{16} \mathrm{~cm}^{-2}$ also presents a high quality single crystalline structure, but a set of filamentary structures known as cellular breakdown are also identified $[35,36]$. In a recent work, the 3D structure and composition of Si cellular-breakdown structures formed in a similar material have been investigated using atom probe tomography [37]. In this work, it has been shown that these filamentary structures are formed by segregation regions of transition metals with up to 
$10 \%$ of Si atomic density. However, these structures are free of silicides, and no evidence of polycrystalline material was observed [37, 38].

To analyze the $\mathrm{V}$ lattice position, we compare the random and channeling spectra of the V-related peak for the two samples. Since there are no noticeable differences in the intensity between the spectra in channeling or random configurations, we can conclude that $\mathrm{V}$ atoms are massively occupying interstitial lattice positions in the host semiconductor. From the comparison of the area of the curves for both, the random and the channeling configurations, we have calculated that the percentage of $\mathrm{V}$ interstitial atoms is $91 \%$ for the sample implanted with $10^{15} \mathrm{~cm}^{-2}$ and $98 \%$ for the sample implanted with $10^{16} \mathrm{~cm}^{-2}$.

Both structural unit cell parameters and V lattice position were also analyzed through DFT quantum calculations. Thus, two different $\mathrm{V}$-implanted configurations were studied in this

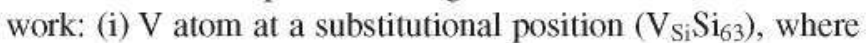
the $\mathrm{V}$ atom replaces one of the $\mathrm{Si}$ atoms in the lattice and (ii) a $\mathrm{V}$ atom at interstitial position $\left(\mathrm{V}_{\mathrm{j}} \mathrm{Si}_{64}\right)$, which is located at the centre of a tetrahedron formed by four $\mathrm{Si}$ atoms. The analysis of theoretical formation energies $\left(E_{\mathrm{f}}\right)$ can be used as a measurement of the stability of positioning at different configurations [16, 17]. Formation energies of both kinds of V implantation were calculated from the total energies derived from the above optimizations as the energy difference between the V-implanted compounds and the sum of the energy of each component. Thus, $E_{\mathrm{f}}$ for $\mathrm{V}$-implanted compound at interstitial position $\left(\mathrm{V}_{\mathrm{i}}\right)$ was defined as:

$$
\begin{gathered}
\mathrm{V}_{\mathrm{i}} \text {-SupersaturatedSilicon }\left(\mathrm{V}_{\mathrm{i}} \mathrm{Si}_{64}\right): \\
E_{\mathrm{f}}=E_{\mathrm{V}_{\mathrm{j}} \mathrm{Si}_{64}}-\left(E_{\mathrm{Si}_{64}}+1 / 2 E_{\mathrm{V}_{2}}\right)=0.97 \mathrm{eV} .
\end{gathered}
$$

Here, $E_{\mathrm{Si}_{64}}, E_{\mathrm{V}_{1} \mathrm{Si}_{64}}$ and $E_{\mathrm{V}_{2}}$ stand for the total energies of bulk $\mathrm{Si}$ (modeled as a $2 \times 2 \times 2$ supercell containing 64 atoms, $\left.\mathrm{Si}_{64}\right), \mathrm{V}$-supersaturated silicon at interstitial position $\left(\mathrm{V}_{\mathrm{i}} \mathrm{Si}_{64}\right)$ and BCC crystalline structure of Vanadium, respectively. In the case of the substitutional positioning, the formation energy corresponds to:

$$
\begin{gathered}
\mathrm{V}_{\mathrm{Si}^{-}} \text {Supersaturated Silicon }\left(\mathrm{V}_{\left.\mathrm{Si}_{\mathrm{Si}} \mathrm{Si}_{63}\right)}:\right. \\
E_{\mathrm{f}}=E_{\mathrm{V}_{\mathrm{Si}_{\mathrm{S}}} \mathrm{Si}_{63}}-\left(63 / 64 E_{\mathrm{Si}_{64}}+1 / 2 E_{\mathrm{V}_{2}}\right)=3.06 \mathrm{eV},
\end{gathered}
$$

where $E_{\mathrm{V}_{\mathrm{S}_{1}} \mathrm{Si}_{63}}$ stands for the total energy at substitutional position $\left(\mathrm{V}_{\mathrm{Si}} \mathrm{Si}_{63}\right)$. As a result, $\mathrm{V}$-supersaturated $\mathrm{Si}$ with $\mathrm{V}$ at interstitial position $\left(\mathrm{V}_{\mathrm{i}} \mathrm{Si}_{64}\right)$ yields an energy difference $(0.97 \mathrm{eV}$ per $\mathrm{V}$ atom) considerably lower than the substitutional one (3.06 eV per $\mathrm{V}$ atom). Thus, computed formation energies indicate that the interstitial position of a $\mathrm{V}$ atom in bulk $\mathrm{Si}$ is more stable than substitutional positioning. This result is in agreement with the results extracted from our previous analysis of the RBS-c (figure 2), where we observed that $\mathrm{V}$ atoms sit mainly in interstitial positions. Similar results regarding to the preferred position of the transition metal have been also reported by our group for Ti implanted silicon compounds [16].

Based on computed formation energies, the analysis of the main structural parameters, electronic structure and absorption coefficient has been carried out only for the V-interstitial Si

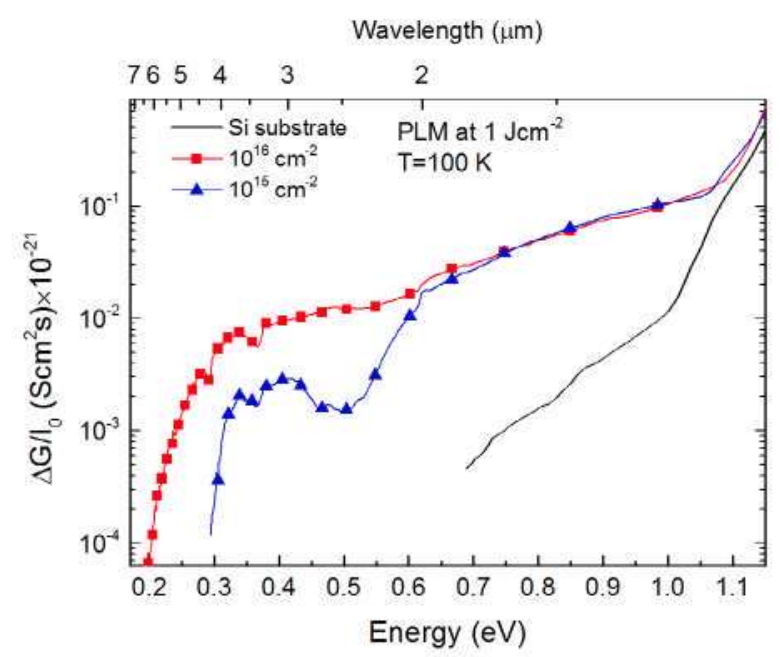

Figure 3. Spectral photoconductance response normalized to the impinging light power density for the Si unimplanted reference sample and for the V implanted Si samples at $10^{15}$ and $10^{16} \mathrm{~cm}^{-2}$ doses. Implanted samples were subsequently PLM processed at $1 \mathrm{~J}$ $\mathrm{cm}^{-2}$. Measurements were carried out at $100 \mathrm{~K}$.

compound. V-supersaturated Si compound with V at interstitial position $\left(\mathrm{V}_{\mathrm{i}} \mathrm{Si}_{64}\right)$ does not lead to important differences in the bulk-Si structure, since both the lattice parameter $a=5.465$ $\AA$ and $d_{\mathrm{Si}-\mathrm{Si}}=2.367 \AA$ (the distance between two Si atoms located in the farthest available positions from the $\mathrm{V}$ atom) are marginally affected with respect to bulk Si ( $a=5.465 \AA$ and $d_{\mathrm{Si}-\mathrm{Si}}=2.366 \AA$ ). Optimized structure indicates that $\mathrm{V}$ atoms are simultaneously coordinated to the four nearest $\mathrm{Si}$ atoms (in a tetrahedrical coordination with a distance equal to $2.440 \AA$ ) and to the six second nearest $\mathrm{Si}$ (in an octahedral configuration with a distance equal to $2.746 \AA$ ). Our previous work, dealing with structural and electronic properties of Ti-supersaturated Si systems through DFT simulations, also exhibited the preference of the transition metal for the interstitial position with a simultaneous tetrahedrical and octahedrical coordination [16].

Spectral photoconductance measurements performed in the $1-7 \mu \mathrm{m}$ wavelength range $(1.15 \mathrm{eV}-0.17 \mathrm{eV})$ give us information about the sub-bandgap photo-generation processes that take place in the V-supersaturated Si material. Figure 3 presents the spectral photoconductance normalized to the impinging light power $\left(\Delta G / I_{0}\right)$ for the samples implanted with $10^{15} \mathrm{~cm}^{-2}$ and $10^{16} \mathrm{~cm}^{-2}$ and subsequently PLM processed with a single $20 \mathrm{~ns}$ pulse at $1 \mathrm{~J} \mathrm{~cm}^{-2}$. Measurements were performed at low temperature $(100 \mathrm{~K})$, in order to increase the signal-to-noise ratio. For a more detailed explanation, see room temperature measurements for similar samples previously published [27, 39]. The silicon unimplanted substrate presents two different behaviours: a slight sub-bandgap photoresponse for photon energies in the $0.7 \mathrm{eV}-1 \mathrm{eV}$ range, as well as an abrupt increase of the photoresponse for energies higher than $1.1 \mathrm{eV}$. The slight sub-bandgap photoresponse would be related to the silicon surface states that create levels in the silicon band gap. These states can be photoexcited with subbandgap light $[40,41]$. The abrupt increase of the photoresponse for photon energies higher than $1.1 \mathrm{eV}$ is related to the VB-CB optical transitions. 


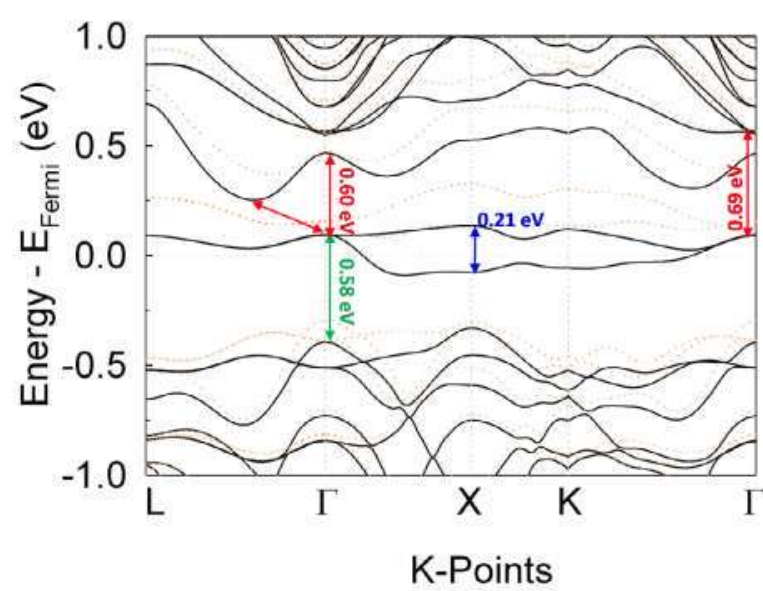

Figure 4. Theoretical band structure for $\mathrm{V}_{\mathrm{i}}$-silicon compound $\left(\mathrm{V}_{1} \mathrm{Si}_{63}\right)$, along main energy differences (corrected according to $G_{0} W_{0}$ approach) between VB-CB (red), VB-IB (green) measured at $\Gamma$ point and IB bandwidth (blue). Solid/dotted line stands for spin up/down channel.

Regarding the V-supersaturated $\mathrm{Si}$ samples, we can observe differences in the spectral photoresponse depending on the implanted dose. Firstly, note that both samples present a photoresponse several orders of magnitude higher than the Si unimplanted substrate for sub-bandgap photons, which is extended far into the infrared region of the spectrum, up to $0.2 \mathrm{eV}(6.2 \mu \mathrm{m})$. Secondly, abrupt edges of the infrared photoresponse are observed at $0.2 \mathrm{eV}$ for the sample implanted with $10^{16} \mathrm{~cm}^{-2}$ and $0.3 \mathrm{eV}$ and $0.55 \mathrm{eV}$ for the sample implanted with $10^{15} \mathrm{~cm}^{-2}$. This photoresponse can be related neither to defects associated with the ion implantation nor to the PLM process $[42,43]$.

As we have recently shown [25], these V-supersaturated Si samples have electrical transport properties that confirmed the insulator-to-metal transition and the formation of an IB in a high quality material. Since spectral photoconductance is directly related to the absorption coefficient [44], we suggest that the intense sub-bandgap photoresponse of the V supersaturated Si samples, together with the presence of abrupt photoresponse edges is consistent with optical transitions from the VB to the IB and from the IB to the CB. In fact, the two abrupt photoresponse edges at $0.3 \mathrm{eV}$ and $0.55 \mathrm{eV}$ of the sample implanted with $10^{15} \mathrm{~cm}^{-2}$ could be signatures of each one of the optical sub-bandgap transitions involving the IB. The sample implanted with the highest dose of $10^{16} \mathrm{~cm}^{-2}$ presents just one abrupt edge of the photoresponse at $0.2 \mathrm{eV}$ and a slight shoulder is observed around $0.65 \mathrm{eV}$. In a previous work [42], the sub-bandgap photoresponse of a similar sample was analysed, and the abrupt edge of the photoresponse at $0.2 \mathrm{eV}$ was already related with an optical transition between the IB and one of the classical bands of the semiconductor (VB or $\mathrm{CB}$ ). This is now modified according to the theoretical calculations here presented. We suggest that the fact that we can only identify one abrupt edge of the photoresponse, instead of two as for the sample implanted with $10^{15} \mathrm{~cm}^{-2}$, could be related with the thicker layer of IB material presented in this sample. A thicker layer of IB material would increase the optical absorption up to a point that individual optical transitions overlap. As a consequence, the higher energy optical transition appears as a slight shoulder at $0.65 \mathrm{eV}$.

To explain the spectral photoresponse shown in figure 3 , a detailed analysis of the electronic band structure of $\mathrm{V}_{\mathrm{i}^{-}}$ supersaturated compoundsis presented. For that, precise quantum calculations based on many-body perturbation theory in $G W$ (see theoretical methods) approximation have been carried out to obtain accurate values for the bandgap energy. In addition, we have also assessed the optical properties through the calculation of the imaginary part of the dielectric function (which is easily related to the absorption coefficients). Note that the formation of defects (mainly Si vacancies) is inevitable with the ion implantation and PLM processes [45, 46]. As matter of fact, several models of V-supersaturated $\mathrm{Si}$ at interstitial positions along to one $\mathrm{Si}$ vacancy $\left(\mathrm{V}_{\mathrm{i}} \mathrm{Si}_{63}\right)$ randomly localized were also optimized, focusing our attention on the optimized geometry with the lowest total energy, whose $E_{\mathrm{f}}=-1.30 \mathrm{eV}$. In consequence, the $\mathrm{V}_{\mathrm{i}}$ implantation in presence of $\mathrm{Si}$ vacancies would lead to a process energetically favored. We are aware that the quantity of Si vacancies considered in our DFT calculations could be higher than those present in experimental samples. Therefore, formation energy defined for $\mathrm{V}_{\mathrm{i}} \mathrm{Si}_{63}\left(E_{\mathrm{f}}=-1.30 \mathrm{eV}\right)$ could be only extrapolated to those situations wherein the vacancy is sufficiently far from the $\mathrm{V}$ atom, whose topology should be similar than those described by DFT optimized structure. Since only $\mathrm{V}_{\mathrm{i}} \mathrm{Si}_{63}$ provides an energetic favored process, photoconductance experiments (figure 3 ) have been interpreted based on results obtained for $\mathrm{V}_{\mathrm{i}} \mathrm{Si}_{63}$. Figures 4 and 5 show the electronic band structure and the imaginary part of the dielectric function computed for $\mathrm{V}_{\mathrm{i}}$-supersatured $\mathrm{Si}$ compound at interstitial positionwith one $\mathrm{Si}$ vacancy $\left(\mathrm{V}_{\mathrm{i}} \mathrm{Si}_{63}\right)$.

The electronic band structure plotted in figure 4 is characterized by the presence of an intermediate band located into the gap of the host semiconductor, whose bandwidth is equal to $0.21 \mathrm{eV}$. In particular, such IB is due to the low energy $t_{2 \mathrm{~g}}$ triplet for spin up. Similarly to the $\mathrm{V}_{\mathrm{i}}$-supersaturated compound without vacancies, each $\mathrm{V}$ atom is simultaneously coordinated to the four nearest $\mathrm{Si}$ atoms and the six second nearest $\mathrm{Si}$. Thus, this octahedral coordination splits $3 d$ levels of $\mathrm{V}$ atoms into a low energy $t_{2 \mathrm{~g}}$ triplet (which plays as IB for spin up channel, while its energy is larger than Fermi level for spin down) and a high-energy $e_{\mathrm{g}}$ doublet (located over the Fermi level for both spin up and spin down channels). We note that at $\Gamma$ point, VB-CB energy differences due to the occupied and empty bands of the host semiconductors are increased up to $1.27 \mathrm{eV}$ and $1.12 \mathrm{eV}$ for spin up and down components with respect to bulk $\mathrm{Si}$. For the spin up component, the IB yields an energy difference of $0.58 \mathrm{eV}$ with the $\mathrm{VB}$, while the energy difference with the $\mathrm{CB}$ of the host semiconductors is $0.69 \mathrm{eV}$. The energy difference calculated between partially filled $t_{2 \mathrm{~g}}$ triplet and empty $e_{\mathrm{g}}$ doublet is equal to $0.60 \mathrm{eV}$. The two latter energy differences would be related with IB-CB transitions. Besides, empty $e_{\mathrm{g}}$ doublet also allows transitions from the VB of the host semiconductor (labeled as VB-CB) with energy of $1.18 \mathrm{eV}$. Regarding the spin down component, in addition to VB-CB coming from the host semiconductors, the empty 


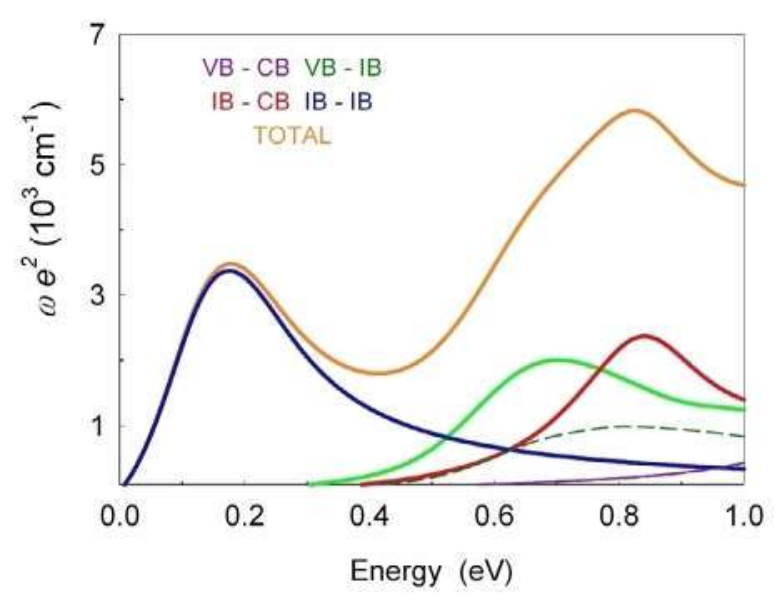

Figure 5. Imaginary part of the dielectric functions calculated for V-supersaturated compounds at interstitial positions in presence of $\mathrm{Si}$ vacancies $\left(\mathrm{V}_{\mathrm{i}} \mathrm{Si}_{63}\right)$, for partial electronic contributions below $1.0 \mathrm{eV}$.

$t_{2 \mathrm{~g}}$ triplet should also enable transitions from the VB with an energy difference equal to $0.73 \mathrm{eV}$ and $1.07 \mathrm{eV}$.

The decomposition of the imaginary part of the dielectric functions as the sum of its various contributions has demonstrated its suitability to verify the role of the new bands coming from the implanted atom on spectral photoresponse in doped Si samples [16, 17]. As seen in figure 5, in the region below $1.0 \mathrm{eV}$, the contribution of the imaginary part of the dielectric function to the absorption of $\mathrm{V}_{\mathrm{i}}$-supersaturated compound with silicon vacancies $\left(\mathrm{V}_{\mathrm{i}} \mathrm{Si}_{63}\right)$ is characterized by two transitions across the IB appearing at $0.20 \mathrm{eV}$ and $0.80 \mathrm{eV}$, which are due to IB-IB transitions and to the sum of IB-CB transition (at $0.84 \mathrm{eV}$ ) and VB-IB transition at $(0.67 \mathrm{eV}$ ). Even though the peak calculated at $0.80 \mathrm{eV}$ would be slightly overestimated with respect to experimental measurements, it could be associated with the photoresponse measured at $0.55 \mathrm{eV}$ for the sample implanted with $10^{15} \mathrm{~cm}^{-2}$ or $0.65 \mathrm{eV}$ for the samples implanted with $10^{16} \mathrm{~cm}^{-2}$. IB-IB transitions cannot be measured in photoconductance experiments, because they are internal transitions that do not generate free electron-hole pairs. Finally, based on electronic band structure, the abrupt photoresponse edge at $0.30 \mathrm{eV}$ or $0.20 \mathrm{eV}$ experimentally measured for samples implanted with $10^{15} \mathrm{~cm}^{-2}$ or $10^{16} \mathrm{~cm}^{-2}$ doses could be due to indirect IB-CB transitions that provide the lowest IB-CB energy differences. These observations support our main conclusion, that the infrared photoresponse is directly related to optical transitions including the IB.

\section{Conclusions}

In summary, we have combined experimental measurements and quantum mechanical calculations to study the structural and sub-bandgap absorption properties of V-supersaturated $\mathrm{Si}$ material and its potential application as an IB material. Rutherford backscattering spectroscopy (RBS) experiments on V-supersaturated Si samples with a high crystalline quality show that $\mathrm{V}$ mainly occupies interstitial positions. Model systems of bulk $\mathrm{Si}$ and $\mathrm{V}$-supersaturated $\mathrm{Si}$ (where one $\mathrm{V}$ atom is located at substitutional or interstitial position) were fully relaxed through density functional theory (DFT). Computed formation energies also indicate that the interstitial position of the $\mathrm{V}$ atom in bulk $\mathrm{Si}$ is the most favorable. Spectral photoconductance measurements of V-supersaturated $\mathrm{Si}$ samples performed in the $1-7 \mu \mathrm{m}$ wavelength range $(1.15-0.17 \mathrm{eV})$ present a photoresponse several orders of magnitude higher than the Si unimplanted substrate for sub-bandgap photons. This photoresponse is extended far into the infrared region $(\approx 0.2 \mathrm{eV}$ or $6.2 \mu \mathrm{m})$, and its profile is characterized by two abrupt edges observed close to $0.2 \mathrm{eV}$ and $0.6 \mathrm{eV}$. The theoretical study (based on DFT and many-body perturbation in $G W$ approximation) of electronic band structure and absorption coefficient of V-supersaturated compounds at interstitial position highlighted the need to additionally include defects (mainly $\mathrm{Si}$ vacancies) to describe such a profile. Thus, the electronic band structure of $\mathrm{V}$-supersaturated $\mathrm{Si}$ with vacancies is characterized by the presence of an intermediate band (IB) located into the gap of the host semiconductor. The analysis of the dielectric function shows that abrupt photoresponse edges at $\approx 0.2 \mathrm{eV}$ and $\approx 0.6 \mathrm{eV}$ experimentally measured can be rationalized based on the presence of an intermediate band. The former should be due to IB-CB, while the latter is due to the sum of VB-IB and IB-CB transitions. More generally, our combined experimental and theoretical approaches suggest that V-supersaturated Si could be an effective IB material in the implementation of a third generation of photovoltaic devices.

\section{Experimental and Theoretical Methods}

\subsection{Samples fabrication and characterization techniques}

Single-crystal squared samples $1 \times 1 \mathrm{~cm}^{2}$ in size of n-type Si (111) with a thickness of $300 \mu \mathrm{m}(\rho \approx 200 \Omega \mathrm{cm}$; $\mu \approx 1500 \mathrm{~cm}^{2} \mathrm{~V}^{-1} \mathrm{~s}^{-1} ; \quad n \approx 2.2 \times 10^{13} \mathrm{~cm}^{-3}$ at room temperature) were implanted in a refurbished VARIANCF3000 Ion Implanter at $32 \mathrm{keV}$ with ${ }^{51} \mathrm{~V}^{+}$at 2 different doses $\left(10^{15}\right.$ and $10^{16} \mathrm{~cm}^{-2}$ ) using a $7^{\circ}$ tilt angle. Ion implantations were performed at the CAI de Técnicas Físicas of the Universidad Complutense de Madrid (Madrid, Spain). After implantation, we performed a laser melting process known as pulsed laser melting (PLM). PLM is the most recommended process in order to recover the crystalline structure after ion implantation, thus allowing for a higher solute trapping of $\mathrm{V}$ in the $\mathrm{Si}$ lattice [47]. All the samples were PLM processed at $1 \mathrm{~J} \mathrm{~cm}^{-2}$ with a $\mathrm{KrF}$ excimer laser $(248 \mathrm{~nm})$ with a $20 \mathrm{~ns}$ pulse duration at IPG Photonics (New Hampshire, USA).

RBS measurements were carried out at the 5 MV CockroftWalton tandetron accelerator of the Centro de Micro-Analisis de Materiales (CMAM) in the Universidad Autónoma de Madrid. The vacuum of the chamber was $5 \times 10^{-6} \mathrm{~Pa}$. For the measurements, $2 \mathrm{MeV} \mathrm{He}^{+}$ions were used. The scattered ions were detected simultaneously by two $\mathrm{Si}$ semiconductor particle detectors, placed at scattering angles of $170^{\circ}$. The measurements were performed with samples oriented at random and channeling geometry in the direction (111). Numerical simulations of the RBS spectra were obtained using a standard backscattering simulation package (SIMNRA) [32]. 
The spectral photoconductance of the samples for energies below the bandgap was measured using the van der Pauw set up. For this purpose, $100 \mathrm{~nm}$ Ti plus $200 \mathrm{~nm}$ Al triangular contacts were deposited on the four corners of $1 \times 1 \mathrm{~cm}$ square samples by means of the e-beam evaporation technique. With this setup, a fixed current was injected through two adjacent contacts and the differential AC voltage generated over the opposite two contacts was measured while a monochromatic chopped light impinged the samples. A TMc 300 Bentham monochromator with a $\mathrm{SiC}$ filament source was used as infrared monochromatic source. The intensity of the light was calibrated with a Bentham pyrometric detector. Measurements were carried out at $100 \mathrm{~K}$ in order to reduce thermal generation of charge carriers and increase the signal-to-noise ratio. For that, samples were placed inside a closed-cycle Janis cryostat and a vacuum pump was used to avoid moisture condensation at low temperature. The van der Pauw set up was feed with $1 \mathrm{~mA}$ DC current to avoid self-heating effects. Photovoltage measurements were carried out with a SR830 digital signal processing lock-in amplifier manufactured by Stanford Research Systems. From the photovoltage measurements we extracted values for the photoconductance of the samples normalized to the impinging light power $\left(\Delta G / I_{0}\right)[44]$.

\subsection{Theoretical methods}

The unit cell of the silicon model system was described as a supercell $2 \times 2 \times 2$ of $\mathrm{Si}_{8}$ cubic unit cell (i.e. 64 atoms, labeled as $\mathrm{Si}_{64}$ ). Structures obtained from $\mathrm{Si}_{64}$ due to the addition of $\mathrm{V}$ atoms and/or $\mathrm{Si}$ vacancies were relaxed to study the effects of introducing $\mathrm{V}$ atoms and $\mathrm{Si}$ vacancies on structural and electronic properties. In this way, one $\mathrm{V}$ atom leads to a concentration $[\mathrm{V}] \gg 10^{20} \mathrm{~cm}^{-3}$, which clearly exceeds the Mott limit. Nevertheless, it is expected that the electronic structure for such dilution would elucidate whether an isolated IB can be obtained or if the energy levels from $3 d$ electrons overlap with $\mathrm{VB}$ and/or $\mathrm{CB}$ of bulk Si, despite that the bandwidth of the IB would be slightly increased [16, 17]. Density functional theory (DFT) quantum calculations were carried out using the Perdew, Burke and Ernzerhof (PBE) [48] method, as implemented in the VASP code $[49,50]$. Atomic structures were relaxed using an atomic tolerance of $0.01 \mathrm{eV}^{-1}$. All calculations were done using a $k$-point mesh of $6 \times 6 \times 6$ in the Monkhorst-Pack scheme [51]. A Methfessel-Paxton firstorder scheme with $0.1 \mathrm{eV}$ of smearing was used to extract the occupations of Kohn-Sham eigenvalues [52].

Optical properties were assessed by means of the absorption coefficient derived from the dielectric functions as implemented in VASP code. Thus, the imaginary part was obtained as the sum of independent transitions between Kohn-Sham states, without local field effects, while the real part was obtained from the imaginary part by the Kramers-Krönig relations. To get converged frequency-dependent dielectric properties, approximately 250 bands and an $8 \times 8 \times 8$ sampling of the Brillouin zone were needed. The imaginary part of the dielectric function has been analyzed as a simple sum over independent transitions, which allows separating it into the contributions from different transitions. Accurate many-body calculations were carried out to correct the underestimation of the bandgap. Thus, quasi particle energies were computed for $\mathrm{Si}_{64}$ and $\mathrm{V}$-implanted compounds at the $\Gamma$-point through the $G_{0} W_{0}$ [53], where the self-energy is calculated by the product of Green function $G$ and the screened Coulomb interaction $W$, while DFT eigenvalues and eigenfunctions were used as starting parameters.

\section{Acknowledgments}

The authors would like to acknowledge the CAI de Técnicas Físicas of the Universidad Complutense de Madrid for the ion implantation process and metallic evaporations. This work was partially supported by the Project MADRID-PV (Grant No. 2013/MAE-2780) funded by the Comunidad de Madrid, by the Spanish MINECO (Economic and Competitiveness Ministery) under grants TEC 2013-41730-R and ENE2016-77798C4-4-R and by the Universidad Complutense de Madrid (Programa de Financiación de Grupos de Investigación UCMBanco Santander) under grant 910173-2014.D. The author acknowledges the computer resources and technical assistance provided by the Centro de Supercomputación yVisualización de Madrid (CeSViMa).

\section{ORCID iDs}

Eric Garcia-Hemme ○ https://orcid.org/0000-0001-5328-8341 Gregorio García (• https://orcid.org/0000-0003-3200-3153

\section{References}

[1] Conibeer G et al 2006 Thin Solid Films 511 654-62

[2] Conibeer G 2007 Mater. Today 10 42-50

[3] Luque A and Marti A 2011 Nat. Photon. 5 137-8

[4] Wolf M 1960 Proc. IRE 48 1246-63

[5] Luque A and Martí A 1997 Phys. Rev. Lett. 78 5014-7

[6] Palacios P, Fernández J J, Sánchez K. Conesa J C and Wahnón P 2006 Phys. Rev. B 73085206

[7] Palacios P. Sánchez K, Conesa J C, Fernández J J and Wahnón P 2007 Thin Solid Films 515 6280-4

[8] Palacios P, Aguilera I, Sánchez K, Conesa J C and Wahnón P 2008 Phys. Rev. Lett. 101046403

[9] Ramiro I, Martí A, Antolín E and Luque A 2014 IEEE J. Photovolt. 4 736-48

[10] Hill R and Bendall F A Y 1960 Nature 186 136-7

[11] Rühle S 2016 Sol. Energy 130 139-47

[12] Silvestre S, Boronat A, Molina M, Castañer L, Olea J, Pastor D, del Prado A, Mártil I, González-Díaz G and Luque A 2013 Japan. J. Appl. Phys. 52122302

[13] Lucena R, Conesa J C, Aguilera I, Palacios P and Wahnón P 2014 J. Mater: Chem. A $228236-45$

[14] López N, Yu K M, Tanaka T and Walukiewicz W 2016 Adv. Energy Mater. 61501820

[15] Martí A, Antolín E, Stanley C R, Farmer C D, López N, Díaz P, Cánovas E, Linares P G and Luque A 2006 Phys. Rev. Lett. 97247701

[16] Sánchez K, Aguilera I, Palacios P and Wahnón P 2009 Phys. Rev. B 79165203

[17] Sánchez K, Aguilera I, Palacios P and Wahnón P 2010 Phys. Rev. B 82165201

[18] Olea J, del Prado A, Pastor D, Mártil I and González-Díaz G 2011 J. Appl. Phys. 109113541 
[19] Wahnón P, Conesa J C, Palacios P, Lucena R, Aguilera I, Seminovski Y and Fresno F 2011 Phys. Chem. Chem. Phys. 13 20401-7

[20] Lucena R, Aguilera I, Palacios P, Wahnón P and Conesa J C 2008 Chem. Mater: 20 5125-7

[21] Antolín E, Martí A, Olea J, Pastor D, González-Díaz G, Mártil I and Luque A 2009 Appl. Phys. Lett. 94042115

[22] Luque A, Martí A, Antolín E and Tablero C 2006 Physica B $382320-7$

[23] Castán H, Pérez E, García H, Dueñas S, Bailón L, Olea J, Pastor D, García-Hemme E, Irigoyen $\mathrm{M}$ and González-Díaz G 2013 J. Appl. Phys. 113024104

[24] Olea J, Pastor D, García-Hemme E, García-Hernansanz R, del Prado A, Mártil I and González-Díaz G 2012 Thin Solid Films $5206614-8$

[25] García-Hemme E, Montero D, García-Hernansanz R, Olea J, Mártil I and González-Díaz G 2016 J. Phys. D: Appl. Phys. 49275103

[26] Winkler M T, Recht D, Sher M J, Said A J, Mazur E and Aziz M J 2011 Phys. Rev. Lett. 106178701

[27] García-Hemme E, García-Hernansanz R, Olea J, Pastor D, del Prado A, Mártil I and González-Díaz G 2014 Appl. Phys. Lett. 104211105

[28] Pastor D, Olea J, del Prado A, García-Hemme E, García-Hernansanz R, Mártil I and González-Díaz G 2013 J. Phys. D: Appl. Phys. $\mathbf{4 3} 135108$

[29] Rebohle L, Prucnal S and Skorupa W 2016 Semicond. Sci. Technol. 31103001

[30] Zhou S, Liu F, Prucnal S, Gao K, Khalid M, Baehtz C, Posselt M, Skorupa W and Helm M 2015 Sci. Rep. 58329

[31] Liu F, Prucnal S, Hübner R, Yuan Y, Skorupa W, Helm M and Zhou S 2016 J. Phys. D: Appl. Phys. 49245104

[32] Mayer M 1999 AIP Conf. Proc. 475 541-4

[33] Chong Y F, Pey K L, Lu Y F, Wee A T S and See A 2001 Surf. Rev. Lett. 8 441-5

[34] Tsao J Y, Aziz M J, Thompson M O and Peercy P S 1986 Phys. Rev. Lett. $562712-5$

[35] Sriranganathan R 1983 J. Cryst. Growth 62 265-83
[36] Cullis A G, Hurle D T J, Webber H C, Chew N G, Poate J M. Baeri P and Foti G 1981 Appl. Phys. Lett. 38 642-4

[37] Akey A J, Recht D, Williams J S, Aziz M J and Buonassisi T 2015 Adv. Funct. Mater. 25 4642-9

[38] Pastor D, Olea J, del Prado A, García-Hemme E, Mártil I, González-Díaz G, Ibáñez J, Cuscó R and Artús L 2011 Semicond. Sci. Technol. 26115003

[39] Olea J, López E, Antolín E, Luque A, García-Hemme E, Pastor D, García Hernansanz R, del Prado A and GonzálezDíaz G 2016 J. Phys. D: Appl. Phys. 49055103

[40] Müller W and Mönch W 1971 Phys. Rev. Lett. 27 250-3

[41] Müller W and Mönch W 1974 Phys. Status Solidi A 24 197-206

[42] García-Hemme E, García-Hernansanz R, Olea J, Pastor D, del Prado A, Mártil I and González-Díaz G 2013 Appl. Phys. Lett. 103032101

[43] Olea J, Pastor D, del Prado A, García-Hemme E, García-Hernansanz R, Mártil I and González-Díaz G 2013 J. Appl. Phys. 114053110

[44] García-Hemme E, García-Hernansanz R, Olea J, Pastor D, del Prado A, Mártil I and González-Díaz G 2012 Appl. Phys. Lett. 101192101

[45] Romano L, Piro A M, Privitera V, Rimini E, Fortunato G, Svensson B G, Foad M and Grimaldi M G $2006 \mathrm{Nucl}$. Instrum. Methods Phys. Res. B $25350-4$

[46] Mannino G, Privireta V, La Magna A and Rimini E 2005 Appl. Phys. Lett. 86051909

[47] White C W, Wilson S R, Apleton B R and Young F W Jr 1980 J. Appl. Phys. 51 738-49

[48] Perdew J P and Ernzerhof K B 1996 Phys. Rev. Lett. 773865

[49] Kresse G and Hafner J 1993 Phys. Rev. B 47 558-61

[50] Kresse G and Furthmüller J 1996 Phys. Rev. B $5411169-86$

[51] Monkhorst H J and Pack J D 1976 Phys. Rev. B 13 5188-92

[52] Methfessel M and Paxton A T 1989 Phys. Rev. B $403616-21$

[53] Hedin L 1965 Phys. Rev. 139 A796-823 